\title{
Grundlagen des Arbeitsrechts im frankophonen Afrika
}

VON GUNTER WiedENSOHLER

Hauptzweck des Arbeitsrechts ist die Absicherung des Beschäftigten gegen die Nachteile und Risiken seiner Stellung. Neben diese Schutztendenz, die das Arbeitsrecht zu einem wesentlichen Teil der staatlichen Sozialordnung macht, tritt immer deutlicher als ein weiterer Wesenszug die Mitgestaltung, der Unternehmens- und Wirtschaftsverfassung. Mit seiner Rollenverteilung in den Betrieben und der Lenkung von Erträgen übernimmt das moderne Arbeitsrecht auch der Entwicklungsländer weitgehend wirtschaftsordnende Funktionen. So wird die unternehmerische Entscheidung erheblich beeinflußt durch die kollektive Festlegung von Arbeitsbedingungen und durch Mitwirkungsrechte der Belegschaft. Bei aller ,,systemimmanent" unterschiedlichen Bewertung des Produktionsfaktors Arbeit ist schließlich nicht zu übersehen, daß die staats- und die privatwirtschaftlich orientierten Länder - auch Afrikas - in der rechtlichen Behandlung der Arbeitsbeziehungen weithin übereinstimmen ${ }^{\mathbf{1}}$. Die Ursprünge einer obrigkeitlichen Regelung der unselbständigen Erwerbstätigkeit im frankophonen Afrika reichen weit zurück in die Epoche der Kolonialherrschaft. Bis zur Schaffung eines einheitlichen Arbeitsgesetzbuches für alle französischen Ubersee-Territorien im Jahre 1952 wurde diese Materie von einer hierarchisch gegliederten Exekutive gestaltet $^{2}$. Wichtigste Quellen des Arbeitsrechts waren die Dekrete des Präsidenten der Republik, vorgelegt durch den Kolonialminister, sowie die Verordnungen und Runderlasse der Gouverneure ${ }^{3}$. Die Kodifikationen der heutigen souveränen Staaten sind, wie noch zu sehen sein wird, maßgebend geprägt durch die kolonialrechtliche Hinterlassenschaft der Franzosen.

\section{Frühere gesetzliche Regelungen}

Am Anfang der Entwicklung eines Arbeitsrechts stand die Uberwindung der Sklaverei, die in Französisch-Westafrika endgültig erst durch ein Regierungsdekret aus dem Jahre 1920 abgeschafft wurde ${ }^{4}$. Das Verbot des Menschenhandels bedeutete jedoch nicht zugleich die Emanzipation des afrikanischen Arbeiters. Zur Erschließung und damit besseren wirtschaftlichen Nutzung des Landes nahmen die Kolonisatoren noch lange Zeit das Recht für sich in Anspruch, Eingeborene zur Zwangsarbeit heranzuziehen (travail forcé), die nicht selten ohne Entgelt erfolgte oder ausschließlich mit Sachleistungen vergütet wurde. Später beschränkte man sich auf die weniger rigorose ,Dienstverpflichtung“ (travail obligatoire), bei der dem Betroffenen hinsichtlich der Art und Einteilung seiner Tätigkeit ein gewisser Spielraum verblieb ${ }^{5}$. Erst das Ende des zweiten Weltkrieges brachte neben anderen Liberalisierungen auch die Aufhebung dieser öffentlich-rechtlichen Verpflichtung des westafrikanischen Arbeiters 6 .

1 Vgl. Wolfgang Zöllner, Arbeitsrecht, München 1977, S. 1-12.

2 P.-F. Gonidec/Martin Kirsch, Le droit du travail des territoires d'outre-mer, Paris 1958, S. 46; Pierre Monnet, La législation du travail dans les colonies françaises, Paris 1925, S. 31.

3 Der Generalgouverneur für Französisch-Westafrika mit Sitz in Dakar war Repräsentant und Treuhänder der Pariser Regierung.

4 Dekret vom 8. 8. 1920: Journal Officiel de la République Française vom 20. 8. 1920.

5 Paul Dareste/M. Sambuc, Traité de droit colonial, Paris 1932, S. 541.

6 Erik Ehrentraut, Die Entwicklung des Arbeitsrechts im ehemaligen Französisch-Westafrika, Hamburg 1973, S. 10. 


\section{Individualarbeitsrecht}

Als erste zusammenhängende ,,Regelung der Eingeborenenarbeit“ gilt das Dekret vom 22. Oktober $1925^{7}$, das neben der Einführung eines schriftlichen Arbeitsvertrages weitere Maßnahmen vorsah, die eine rücksichtlose Ausnutzung des ehedem praktisch ungeschützten Arbeitnehmers verhindern sollten. Dazu gehörten die amtliche Beglaubigung des Anstellungskontraktes, erforderlichenfalls dessen Ubersetzung in den Heimatdialekt des Beschäftigten, Strafdrohungen für verschiedene Zuwiderhandlungen des Arbeitgebers, die Errichtung einer behördlichen Arbeitsinspektion und die Konstituierung von Schiedsräten, die dem Beschäftigten die kostenlose Durchsetzung seiner vertraglichen Ansprüche ermöglichen sollten. Wichtige Einzelheiten wie etwa die Festsetzung von Mindestlöhnen blieben den Ausführungsverordnungen der Gouverneure vorbehalten ${ }^{8}$, derenErlaß sich jedoch vielfach um Jahre verzögerte, so daß die soziale Stellung des antochthonen Arbeiters sich zunächst kaum merklich verbesserte. Zudem nutzten nur relativ wenige Stellenbewerber die ihnen gebotene Möglichkeit eines schriftlichen Vertragsschlusses. Damit verbunden war nämlich regelmäßig die Ausstellung eines Arbeitsbuches, dessen Kontrollfunktion das verständliche Mißtrauen des Afrikaners erregte, mußte er doch jederzeit seine Einberufung zur Zwangsarbeit befürchten ${ }^{9}$.

Zu Beginn des Jahres 1932 wurde die im Mutterland geltende gesetzliche Regelung von Arbeitsunfällen mit einigen Änderungen auf Westafrika ausgedehnt ${ }^{\mathbf{1 0}}$. Bei ernsteren Verletzungen, die unmittelbar auf die Beschäftigung zurückzuführen waren, erhielt der ohne eigene Schuld Betroffene oder sein Rechtsnachfolger einen Entschädigungsanspruch gegen den Arbeitgeber. Die Höhe der als Rente zu gewährenden Ersatzleistung hat sich danach bemessen, ob der Unfall zu einer dauernden (vollständigen oder teilweisen) Invalidität, zu einer vorübergehenden Arbeitsunfähigkeit oder zum Tode des Beschäftigten führte. Der mit allen entstehenden Kosten - für Heilbehandlung, Medikamente usw. - belastete Arbeitgeber konnte sich von seinen Verpflichtungen ganz oder teilweise befreien, indem er die Beschäftigten bei einer anerkannten Ersatzkasse versicherte. Die Reichweite der Regelung war ziemlich begrenzt, da die große Masse der Saison- und Hilfsarbeiter wie generell die in der Landund Forstwirtschaft Tätigen nicht erfaßt wurden. Zudem galten nur solche Mißgeschicke als Arbeitsunfall, die sich beim Gebrauch einer mit mechanischer Kraft betriebenen Maschine ereigneten ${ }^{11}$. Alle anderen, den strengen Voraussetzungen dieses Dekretes nicht genügenden Unfälle und Berufskrankheiten unterlagen einer älteren Verordnung, die eine am Grad des Mitverschuldens orientierte, durch den Gebietsgouverneur jeweils festzusetzende Abfindung vorsah ${ }^{12}$.

Ein 1936 erlassenes Dekret ${ }^{13}$ traf Vorkehrungen eigens zum Schutze berufstätiger Kinder, Jugendlicher und Frauen. Die Beschäftigung von Kindern unter 14 Jahren war mit Ausnahme der Familienarbeit fortan grundsätzlich verboten; die Arbeit von Jungen unter 15 und Mädchen unter 17 Jahren durfte eine bestimmte Dauer nicht überschreiten und mußte von Ruhetagen und angemessenen Pausen unterbrochen sein. Frauen durften acht Wochen vor

\footnotetext{
7 Journal Officiel de l'Afrique Occidentale Française vom 3. 4. 1926.

$8 \mathrm{Vgl}$. Orde Brown, The African Labourer, 2. Aufl. London 1967, S. $184 \mathrm{ff}$

9 Dazu Pierre Pélisson, Les problèmes du travail, in: Afrique Occidentale Française, Bd. I (Ed. Guernier), Paris 1949, S. 312.

10 Dekret vom 2. 4. 1932: Journal Officiel de la République Française vom 8. 4. 1932; dazu E. Ehrentraut, a. a. O., S. 17 ff.

11 Jean Gorce, Les accidents du travail dans les territoires français d'Afrique, in: Revue juridique et politique de l'Union Francaise 5 (1951), S. 177

12 P. Dareste/M. Sambuc, a. a. O., S. 573.

13 Dekret vom 18. 9. 1936: Journal Officiel de la République Française vom 20. 9. 1936; Durchführungsverordnung: Journal Officiel de l'Afrique Occidentale Française vom 29. 5. 1937.
} 
und nach der Niederkunft der Arbeit fernbleiben und waren während einer Zeit von 18 Monaten nach der Geburt berechtigt, zweimal täglich eine Stillpause von je 20 Minuten einzulegen.

\section{Kollektives Arbeitsrecht}

Schon bald wurde den Arbeitnehmern das Recht zugestanden, sich zum Zwecke der Wahrnehmung ihrer Interessen in Gewerkschaften zusammenzuschließen ${ }^{\mathbf{1 4}}$. Darunter begriff man rechts- und prozeßfähige Vereinigungen von Angehörigen gleichartiger Berufe, die der Leitung durch französische Staatsbürger und der Aufsicht durch die oberen Verwaltungsbehörden unterstanden. Von der Koalitionsfreiheit war diese Regelung noch weit entfernt, konnten doch nur Erwerbstätige mit einem bestimmten, vor allem durch französische Sprachkenntnisse nachzuweisenden Bildungsniveau ungehindert Mitglied werden. Für alle anderen Afrikaner, d. h. die Masse der arbeitenden Bevölkerung, war der Beitritt zu einer Gewerkschaft zum Teil erheblich erschwert ${ }^{15}$. Jederzeit frei stand den Arbeitnehmern dagegen der Zusammenschluß in Berufsverbänden ohne eigene Rechtspersönlichkeit mit einem auf die Belange der jeweiligen Tätigkeitsart begrenzten Aktionsradius.

Zugleich erging eine umfangreiche Regelung des Tarifvertragsrechts, dessen Geltung allerdings beschränkt war auf die Arbeitsbeziehungen in Industrie, Handel und Transportgewerbe ${ }^{\mathbf{1 6}}$. Der zwischen Gewerkschaft und Arbeitgeberverband (oder einzelnen Unternehmen) auf Zeit oder für die Dauer eines bestimmten Vorhabens abgeschlossene Tarifvertrag behandelte innerhalb seines räumlichen Geltungsbereichs $u$. a. Fragen der Entlohnung, der Mitbestimmung, der Kündigung und der Streitentscheidung. Durch Verfügung des Gouverneurs, die im Gesetzblatt zu veröffentlichen war, konnte der Vertrag für allgemeinverbindlich erklärt werden, wodurch sich seine Normen auf die nicht tarifgebundenen Arbeitgeber und Arbeitnehmer der betreffenden Branche erstreckten.

Die Einführung von Gewerkschaften, Berufsverbänden und Tarifverträgen wäre unvollständig gewesen ohne die gleichzeitig erfolgte ,Regelung von Arbeitskonflikten in Französisch-Westafrika"17. Danach waren Streik und Aussperrung illegal, solange kein Schlichtungsversuch stattgefunden hatte. Das Verfahren richtete sich nach den entsprechenden Vereinbarungen der Tarifpartner. Existierte in dem betroffenen Beschäftigungszweig kein Tarifvertrag, so mußte die zuständige Behörde sich binnen 48 Stunden um eine gütliche Einigung bemühen. Wenn die Beamten den drohenden Arbeitskampf für unverantwortlich hielten, konnten sie nach einem mißlungenen Vermittlungsversuch eineSchiedsinstanz anrufen, deren Entscheidung für alle Beteiligten verbindlich war. Drei Monate Gefängnis und/oder Geldstrafe drohten demjenigen, der diesen Vorschriften zuwider eine gemeinsame Arbeitsniederlegung herbeizuführen suchte oder eine Aussperrung anordnete.

Auch diese Regelungen blieben für viele Einheimische reine Theorie. Zwar kamen in Westafrika bis 1948 immerhin 152 Tarifverträge zustande, etwa $27 \%$ der registrierten Lohnempfänger (salariés), darunter vor allem die Landarbeiter, wurden aber von den Abmachungen nicht erfaßt. Die mit den Tarifverträgen verbundenen Vorteile sollten, so hieß es, allein den

14 Dekret vom 11. 3. 1937: Journal Officiel de la République Française vom 17. 3. 1937.

15 Franz Ansprenger, Politik im Schwarzen Afrika. - Die modernen politischen Bewegungen im Afrika französischer Prägung, Köln und Opladen 1961, S. 219.

16 Dekret vom 20. 3. 1937: Journal Officiel dela République Française vom 24. 3. 1937; Ausführungsbestimmungen: Journal Officiel de l'Afrique Occidentale Française vom 1. 5. 1937.

17 Dekret vom 20. 3. 1937: Journal Officiel de la République Française vom 24. 3. 1937; vgl. E. Ehrentraut, a. a. O., S. 34 f. 
„,weiter entwickelten Arbeitnehmern"18 zugute kommen. Schließlich kam es während des zweiten Weltkrieges und in den Nachkriegsjahren schon deshalb kaum zu Arbeitskonflikten, weil der gewerkschaftliche Einfluß damals noch relativ gering war.

\section{Die Gesetzgebung nach 1946}

Die afrikanische Emanzipationsbewegung erhielt nachhaltigen Auftrieb durch die Konferenz von Brazzaville, auf der im Februar 1944 in Anwesenheit de Gaulles verschiedene Resolutionen gefaßt wurden, die in der Forderung nach mehr Eigenständigkeit und parlamentarischer Vertretung der Afrikaner gipfelten ${ }^{19}$. Unter dem Eindruck dieser politischen Entwicklung hat die Legislative der Nachkriegszeit den Weg einer grundsätzlichen Neuorientierung beschritten, etwa durch Abschaffung der Pflichtarbeit, Einführung einer einheitlichen Währung für die französischen Afrika-Kolonien und Verleihung der französischen Staatsbürgerschaft an die Bewohner aller abhängigen Territorien ${ }^{20}$. Einige Verbesserungen gegenüber den früheren Einzelverordnungen waren in dem ,,Code du travail indegène“ vom 18. Juni $1945^{21}$ für den afrikanischen Arbeitnehmer vorgesehen. Dieses erste überregionale Gesetz zur Regelung der ,Eingeborenenarbeit“ ist jedoch, weil es maßgebenden Kreisen nicht fortschrittlich genug erschien, trotz späterer Korrekturen nie in Kraft getreten ${ }^{22}$. Eine Teilreform des kolonialen Arbeitsrechts gelang dagegen bereits im Jahre 1944, als ein Dekret der provisorischen Regierung de Gaulle die uneingeschränkte Koalitionsfreiheit und die volle Anerkennung der Tariffähigkeit afrikanischer Gewerkschaften brachte ${ }^{23}$.

\section{Der ,Code Moutet“}

Unter der Regie des sozialistischen Úberseeministers Marius Moutet entstand am 17. Oktober 1947 ein mit 167 Artikeln relativ umfangreiches Arbeitsgesetzbuch ${ }^{24}$, das sich von dem gescheiterten „,Code du travail indigène“ in mancher Hinsicht unterschied, gerade deshalb aber schon wenige Wochen nach seiner Verkündung das gleiche Schicksal erlitt. Hatte der „Code Moutet“ mithin nur zeitweilig Geltung erlangt, so ist er dennoch aus folgenden Gründen beachtenswert: Er erstreckte sich erstmals auf sämtliche französischen Kolonien mit Ausnahme Indochinas, galt für alle Arbeitnehmer ohne Ansehung ihres Geschlechts, ihres rechtlichen Status (statut juridique) etc., bildete die Grundlage für den späteren Code du travail von 1952 und zeigte deutlich den Nachkriegswandel in der französischen Afrikapoli$\mathrm{tik}^{25}$.

Das einzelne Arbeitsverhältnis war nach diesem Kodex nur unter Einhaltung einer tarifvertraglich vereinbarten Frist kündbar, die auch bei Aufgabe des Betriebes oder Änderung seiner Rechtsform beachtet werden mußte. Entlassungen unter Mißachtung der Frist führten zu Schadenersatzansprüchen, auf die der Betroffene nicht von vornherein verzichten konnte. Die Möglichkeit des Abschlusses von Tarifverträgen war nicht länger auf einige Wirt-

\footnotetext{
18 Ministerialvorlage zum Dekret vom 20. 3. 1937: Journal Officiel de la République Française vom 24. 3. 1937, S. 3466.

19 F. Ansprenger, a. a. O., S. $60 \mathrm{f}$.

20 Virginia Thompson/R. Adloff, French West Africa, London 1958, S. 10, 50, 278.

21 Dekret vom 18. 6. 1945: Journal Officiel de la République Française vom 20. 6. 1945, S. 3719 ff.

22 P.-F. Gonidec, Cours de droit du travail atricain et malgache, Paris 1966, S. 16.

23 Dekret vom 7. 8. 1944: Journal Officiel de la République Française/Edition Alger vom 17. 8. 1944, S. 720.

24 Journal Officiel de la République Française vom 21. 10. 1947, S. 10402 ff.

25 Vgl. P.-F. Gonidec, L'évolution des territoires d'outre-mer depuis 1946, Paris 1958, S. 20.
} 
schaftszweige beschränkt; auch konnten die Vereinbarungen sich auf weitere Sachbereiche erstrecken und so z. B. Vorschriften enthalten über die Personalvertretung und Lehrlingsausbildung, über Leistungsprämien und Gefahrenzulagen.

Die Regelung der Löhne und Gehälter, für die jeweils Mindestbeträge festgesetzt wurden, stand unter der Maxime ,,gleiche Arbeit, gleicher Lohn“. Einen bestimmten Vergütungszuschlag (indemnité de dépaysement) erhielten die in der Landwirtschaft Westafrikas eingesetzten Wanderarbeiter, sofern die Lebensbedingungen ihres Beschäftigungsortes und ihres gewöhnlichen Aufenthaltsortes erheblich voneinander abwichen ${ }^{26}$. Die Auszahlung hatte an einem Werktag auf der Arbeitsstelle in bar zu erfolgen; eine Bezahlung mit Naturalien war unzulässig. Gehälter wurden einmal im Monat ausgezahlt, Löhne im Abstand von 16 Tagen; bei Akkordarbeiten, die länger als zwei Wochen dauerten, konnte der Zahlungsmodus grundsätzlich frei vereinbart werden. Jeder Arbeitnehmer erhielt regelmäßig eine Lohnbzw. Gehaltsaufstellung. Standen für den letzten Abrechnungszeitraum noch Ansprüche aus, so waren sie im Konkurs des Arbeitgebers bevorzugt zu befriedigen. Mit Ausnahme von Steuern und Beiträgen zur Sozialversicherung waren (freiwillige wie unfreiwillige) Abzüge vom Arbeitsentgelt nur bis zu einem Freibetrag statthaft, dessen Höhe der zuständige Gouverneur festsetzte ${ }^{27}$.

Eine weitere Ausführungsverordnung präzisierte die für Frauen und Kinder verbotenen Tätigkeiten. Schwangere Frauen erhielten vor und nach der Niederkunft je sechs Wochen Urlaub und bezogen während der Arbeitsunterbrechung die Hälfte des bisherigen Lohnes. Jedem Beschäftigten stand pro Woche ein arbeitsfreier Tag zu und pro Jahr ein bezahlter Erholungsurlaub von mindestens 12 Tagen, der nicht durch Geldleistungen kompensierbar war und auf den Arbeitsversäumnisse durch Unfall, Krankheit und Schwangerschaft nicht angerechnet werden durften. Einer dem jeweiligen Gebietsgouverneur unterstellten Arbeitsinspektion oblag die Beratung der Arbeitskräfte in beruflichen Angelegenheiten und die Kontrolle der Durchführung aller sie begünstigenden Rechtsvorschriften. Zur unmittelbaren Vertretung der Belegschaftsinteressen gegenüber dem Unternehmer - etwa in Fragen des Arbeitsschutzes - sah der ,,Code Moutet“ überdies für bestimmte Beschäftigungszweige die Konstituierung von Betriebsräten vor.

Aus dem Arbeitsverhältnis entstehende Streitigkeiten konnten - u. U. kostenlos - vor einen Schiedsrat gebracht werden, der sich aus einem höheren Beamten als Vorsitzenden und jeweils nicht mehr als zwei Arbeitgeber- und Arbeitnehmervertretern zusammensetzte. Die nach geheimer Beratung ergehenden, schriftlich begründeten Schiedssprüche waren nach Ablauf von acht Tagen (eventuell gegen Sicherheitsleistung) vorläufig vollstreckbar und konnten in jedem Fall noch innerhalb eines Monats durch den zuständigen Aufsichtsbeamten der Justizbehörde angefochten werden. Verstöße gegen die Vorschriften des Arbeitsgesetzbuches unterlagen zum Teil empfindlichen Strafen, die im Wiederholungsfall auf das doppelte Maß erhöht werden konnten.

Der ,,Code Moutet“" ist ein sinnfälliger Ausdruck für die auf weitgehende Gleichstellung mit dem Mutterland zielende Assimilationspolitik ${ }^{28}$. Europäische Institutionen und Wertvorstellungen wurden ohne weiteres auf afrikanische Verhältnisse übertragen, obwohl die sozialen Gegebenheiten dort durchaus andere sind. So wurde die gesetzliche Verpflichtung zum Schadenersatz bei Arbeitsflucht möglicherweise nicht dem Afrikaner gerecht, der den Arbeitsplatz auf Anordnung seines Stammesoberhauptes kurzerhand verließ, um in seinem

\footnotetext{
26 Vgl. Elliot P. Skinner, Labor Migration among the Mossi of the Upper Volta, in: Urbanization and Migration in West Africa (Ed. Hilda Kuper), Berkeley 1965, S. $60 \mathrm{ff}$.

27 E. Ehrentraut, a. a. O., S. 42-45.

28 Grundsätzlich Raymond F. Betts, Assimilation and Association in French Colonial Theory, New York 1961.
} 
Heimatort traditionellen Familienpflichten nachzukommen ${ }^{29}$. Grundlegende Unterschiede zwischen Afrikanern und Europäern z. B. hinsichtlich der Arbeitsmoral, der Lebensauffassung und Glaubenshaltung hätten in vielen Fällen eine differenzierende rechtliche Behandlung erfordert ${ }^{30}$.

\section{Der Code du travail von 1952}

Als ein Meilenstein der kolonialrechtlichen Entwicklung ist schließlich die Verabschiedung des Code du travail von $1952^{31}$ durch das französische Parlament zu nennen, eines Arbeitsgesetzbuches, das die Kodifikationen der späteren westafrikanischen Staaten wesentlich beeinflußt hat. Das seinerseits an den ,Code Moutet“ anknüpfende Gesetz verkörperte einen Kompromiß zwischen zwei politischen Richtungen, deren eine den afrikanischen Arbeiter dem des Mutterlandes rechtlich gleichgestellt wissen wollte, während die andere das Ziel einer Anpassung des Rechts an die unterschiedlichen regionalen Bedürfnisse verfolgte ${ }^{32}$. Der Erlaß von Gouverneursverordnungen, die weithin als ein ,,Symbol des Kolonialismus“ gal$\operatorname{ten}^{33}$, wurde fortan erschwert durch eng begrenzte Ermächtigungen und durch die Mitwirkung paritätisch besetzter Konsultativausschüsse. Angesichts der Tatsache, daß im gesamten Geltungsbereich des Gesetzes mehr als 1700 Einzelverordnungen ergingen ${ }^{34}$, hatte diese Rechtsquelle dennoch kaum an Bedeutung verloren. Auf dem Verordnungswege erfolgte $u$. a. eine Kindergeldregelung der Art, daß die entsprechenden Grundsätze des öffentlichen Dienstes mit Wirkung vom 1. Januar 1956 auch für die Privatwirtschaft verbindlich waren. Ermächtigungsgrundlage war Artikel 23 des Arbeitsgesetzbuches, in dem es hieß: ,Die Gouverneure sind berechtigt, nach Konsultierung der Arbeitsberatungsausschüsse und der Gebietsversammlungen - mit Zustimmung des Úberseeministers - Erlasse zu veröffentlichen, durch die für alle Beschäftigten im Sinne dieses Gesetzes Familienzulagen eingeführt sowie Ausgleichskassen für die Zahlung solcher Beihilfen errichtet werden“"35.

Im folgenden sollen einige der durch die Kodifikation eingeführten Neuerungen ihrem wesentlichen Inhalt nach wiedergegeben werden: Einstellungsverträge, die für eine Beschäftigungsdauer von mehr als drei Monaten abgeschlossen wurden, bedurften der Beurkundung durch die zuständige Behörde. Die mündlich erklärte Kündigung war auf Verlangen des betroffenen Arbeitnehmers schriftlich zu bestätigen; willkürliche Entlassungen hatten Ersatzansprüche zur Folge. Lehrverträge enthielten einen ausführlichen Katalog gegenseitiger Rechte und Pflichten und sollten nach Möglichkeit auch in der Muttersprache des Lehrlings abgefaßt sein. Der Tarifvertrag konnte sich auf alle arbeitsrechtlich relevanten Fragen erstrecken, von denen einige (wie Urlaub und Überstunden) zwingend Gegenstand der Úbereinkunft waren. Zum ersten Male wurde die Arbeitszeit auf 40 Wochenstunden begrenzt und darüber hinaus geleistete Arbeit, deren Höchstdauer der Gouverneur festsetzte, als Uberstunden vergütet. Die Rechtsstellung des durch geheime Wahl installierten Betriebsra-

\footnotetext{
$29 \mathrm{Vgl}$. AndréRobert, Le droit du travail et la mentalité africaine, in: Recueil Penant 72 (1962), S. 125 ff.; PierreCharles, Tribal Society and Labour Legislation, in: International Labour Review 67 (1952), S. $425 \mathrm{ff}$.

30 Jean Déprez, Evolution et particularisme du droit de travail en Afrique occidentale, in: Annales Africaines 1960, S. $7 \mathrm{ff}$.

31 Le code du travail dans les territoires d'outre-mer: Journal Officiel de la République Française vom 16. 12. 1952, S. 11 541 ff.

32 Paul Devinat, The Labour Code for French Overseas Territories. - Some Economic Considerations, in: International Labour Review 68 (1953), S. $245 \mathrm{ff}$.

33 P.-F. Gonidec/M. Kirsch, Le droit du travail, a. a. O., S. 53.

34 Lambert Bovy, Aspects historiques et idéologiques du mouvement syndical en Afrique d'expression française, in: Recueil Penant 74 (1964), S. 387.

35 Dazu F. Ansprenger, a. a. O., S. 240.
} 
tes wurde dahin verbessert, daß er die Interessen der Betriebsangehörigen nunmehr auch hinsichtlich der Höhe des Arbeitsentgelts, der beruflichen Einstufung und der tarifvertraglichen Abmachungen wahrnehmen konnte.

Eingehend geregelt war die Austragung arbeitsrechtlicher Konflikte. Bei Kollektivstreitigkeiten, also solchen zwischen Tarifvertragsparteien, traten die Beteiligten unverzüglich unter Vorsitz des Arbeitsinspektors zu Vergleichsverhandlungen zusammen, nach deren Scheitern ein sachverständiger Schlichter einen Einigungsvorschlag unterbreitete. Wenn binnen fünf Tagen keine Partei widersprach, wurde die Stellungnahme des Schlichters amtlich veröffentlicht und damit rechtskräftig. Im Falle eines Widerspruchs urteilte eine Schiedskommission, bestehend aus dem Präsidenten des Appellationsgerichts und zwei Beisitzern, die auf Vorschlag des Arbeitsinspektors vom Gouverneur ernannt wurden.

\section{Weitere Entwicklungen}

Das Gesetzbuch von 1952 bekräftigte die Koalitionsfreiheit und dehnte sie auf eingeborene Frauen aus, deren Eintritt in eine Gewerkschaft damit nicht länger an die Zustimmung des Ehemannes oder Familienoberhauptes gebunden war. In den wenigen Jahren bis 1956 wuchs die Zahl der solcherart organisierten Arbeitskräfte nahezu um das Dreifache auf 166000. Angesichts der Zersplitterung des Gewerkschaftswesens in etwa 500 - untereinander vielfach rivalisierende - Einzelorganisationen hielt der gewerkschaftliche Machtzuwachs sich freilich in Grenzen ${ }^{36}$. Der Code du travail erklärte außerdem nur die bedeutenderen Koalitionen für tariffähig. Dennoch kam es in Westafrika zwischen 1953 und 1955 zu verschiedenen Streiks, die so beachtliche Ergebnisse brachten wie die Realisierung des Kindergeldsystems, der 40-Stunden-Woche und der Entschädigungsregelung bei Arbeitsunfällen ${ }^{37}$. Daneben wurden die Gewerkschaften vor allem aktiv durch ihre Mitsprache in den Betriebsräten, den Schiedsgremien und den Beratungskommissionen für Arbeitsfragen. In der Spätphase der Kolonialära schließlich, beginnend etwa um 1956, lösten die Gewerkschaften sich allmählich aus der (auch organisatorischen) Abhängigkeit von Frankreich und arbeiteten auf einen panafrikanischen Zusammenschluß hin ${ }^{38}$.

Vervollständigt wurde das Úbersee-Arbeitsgesetzbuch durch das Ausführungsdekret vom 24. Februar 1957 betreffend die sozialen Leistungen bei Arbeitsunfällen und Berufskrankheiten ${ }^{39}$. Der Schadensausgleich erfolgte durch die regional zuständige Ersatzkasse, die von dem Unternehmer entsprechende Versicherungsbeiträge erhob. Verunglückte ein Beschäftigter im Betrieb oder auf dem Wege zur Arbeitsstelle, so mußte der Unternehmer die Schadensmeldung in zwei Ausfertigungen an den Arbeitsinspektor leiten, der seinerseits ein Exemplar an die Versicherung weiterreichte. Hatte der Verletzte nicht innerhalb von drei Tagen die Arbeit wiederaufgenommen, so war ein ärztliches Gutachten einzuholen, das Auskunft gab über den Gesundheitszustand des Betroffenen, über die möglichen Folgen seiner Verletzung und die voraussichtliche Dauer der Arbeitsunfähigkeit. Der einklagbare Ersatzanspruch umfaßte sämtliche Behandlungs- und Heilungskosten, die Krankenhaus-, Transport- und Rehabilitationskosten. Gezahlt wurden außerdem Tagegelder für die Zeit der unfallbedingten Arbeitsunterbrechung und gegebenenfalls eine Invaliditäts- bzw. Hinterblie-

36 András November, L'évolution du mouvement syndical en Afrique occidentale, Paris 1965, S. $76 \mathrm{ff}$.

37 P.-F. Gonidec, L'évolution du syndicalisme en Afrique noire, in: Recueil Penant 72 (1962), S. 174; L. Bovy, a. a. O., S. 387.

38 Vgl. Immanuel Geiss, Gewerkschaften in Afrika, Hannover 1965, S. 43.

39 Journal Officiel de la République Française vom 28. 2. 1957, S. 2305 ff. 
benenrente, deren Höhe von einem Beschluß der gesetzgebenden Versammlung des jeweiligen Territoriums abhing. Die zur Regelung von Arbeitsunfällen erlassenen Vorschriften galten entsprechend für die gesetzlich anerkannten Berufskrankheiten und deren Folgen.

\section{Die geltenden Arbeitsgesetzbücher}

Nach Erlangung der Unabhängigkeit haben die afrikanischen Staaten französischer Prägung den Kodex von 1952 durch nationales Recht ersetzt. Als erstes dieser Länder gab Guinea sich am 30. Juni 1960 ein eigenes. Arbeitsgesetzbuch, zeitlick zuletzt die Republik Togo am 8. Mai 197440. Entscheidende Zugeständnisse sind in den geltenden afrikanischen Verfassungen verankert, so z. B. das Recht auf Arbeit und Urlaub, die Koalitionsfreiheit, das Streikrecht und die Abschlußfreiheit bei Arbeitsverträgen. In der Regel wird daneben auch die Pflicht zur Arbeit betont ${ }^{41}$, zugleich aber das Verbot jeder Form von Zwangsarbeit bekräftigt.

Zur Abgrenzung ihres Geltungsbereichs definieren die Gesetze den Arbeitnehmer (travailleur) als eine Person, die ihre beruflichen Dienste einer anderen - natürlichen oder juristischen - Person gegen Bezahlung unterstellt. Für den Arbeitnehmerstatus charakteristisch ist also neben der Entlohnung vor allem die persönliche Abhängigkeit, die Subordination unter die einseitige Weisungsbefugnis des Arbeitgebers, deren Reichweite sich aus der Eigenart der geleisteten Dienste ergibt. Eine rechtliche Sonderstellung nehmen die Beamten ein, d. h. die in der öffentlichen Verwaltung auf Dauer Beschäftigten.

Unabhängig vom Ort des Vertragsschlusses und vom Wohnsitz der einen oder anderen Partei - so lautet eine weithin einheitliche Vorschrift - ist grundsätzlich das Recht des afrikanischen Staates maßgebend, in dessen Gebiet der Arbeitsvertrag durchgeführt wird ${ }^{\mathbf{4 2}}$. Erstreckt die Durchführung des Kontraktes sich auf mehrere Länder, so erklären Madagaskar, Gabun, Tschad etc. ihr nationales Arbeitsrecht allerdings nur für anwendbar, wenn die vereinbarte Dauer der Inlandsbeschäftigung drei (im Falle Kameruns sechs) Monate übersteigt. Gegenstand der folgenden Ausführungen sind zwei Kernbereiche der Gesetzgebung, die schon im Code du travail von 1952 einen Schwerpunkt bildeten: die Vertragsbeziehungen des einzelnen Beschäftigten zu seinem Arbeitgeber (1) sowie Zustandekommen, Inhalt und Wirkungsweise des Tarifvertrages (2).

\section{Der Arbeitsvertrag (contrat de travail)}

Die Gesetzgeber halten am Grundsatz der Vertragsfreiheit fest, verfügen aber eine Reihe von Ausnahmen, sei es unmittelbar durch Gesetz zum Schutze von Leben und Gesundheit des Arbeitnehmers (so die Beschränkungen der Kinder-, Frauen- und Nachtarbeit), sei es mittelbar durch Ermächtigung der Exekutive, etwa aus strukturpolitischen Erwägungen in den Arbeitsmarkt einzugreifen. Die einzelnen Regeln weichen in dieser Hinsicht meist nur geringfügig voneinander ab. Durchweg beibehalten wird auch die Höchstdauer von Zeitverträgen und das Verbot der Einstellung auf Lebenszeit. Vertragliche Arbeitsverbote in Gestalt von Konkurrenzklauseln sind nur wirksam, wenn das Beschäftigungsverhältnis durch ein

\footnotetext{
40 Ein Verzeichnis der amtlichen Fundstellen aller einschlägigen Gesetze findet sich bei Martin Kirsch, Le droit du travail africain Bd. I, Paris 1975, S. 337-341.

41 Näheres bei Enno Kliesch, Der Einfluß des französischen Verfassungsdenkens aú afrikanische Verfassungen, Hamburg 1967, S. 73 f. 42 So bereits der Code du travail von 1952; vgl. Cour suprême Dahomey 19. 4. 1964: Travail et profession d'outre-mer Nr. 195 , S. 4317.
} 
vorwerfbares Verhalten des Arbeitnehmers beendet worden ist. Außerdem muß das Konkurrenzverbot örtlich und zeitlich begrenzt bleiben, z. B. im Falle Senegals auf zwei Jahre und auf einen Umkreis von $200 \mathrm{~km}$ vom bisherigen Beschäftigungsort. Eine weitere Beschränkung der Vertragsfreiheit bringt das Arbeitsgesetzbuch der Republik Mali, indem es die Einstellung von Ausländern an eine behördliche Genehmigung knüpft ${ }^{\mathbf{4 3}}$.

\section{a) Dauer und Inhalt des Arbeitsvertrages}

Die Gesetzgebung unterscheidet hinsichtlich der Beschäftigungsdauer zwischen zeitlich begrenzten Verträgen, zeitlich unbegrenzten Verträgen und solchen, die auf Probe abgeschlossen werden. Für einen bestimmten oder bestimmbaren Zeitraum eingegangene Arbeitsverhältnisse, zu denen in Senegal und Mauretanien ausdrücklich auch die projektgebundene Beschäftigung rechnet, dürfen durchweg eine Dauer von zwei (Madagaskar drei) Jahren nicht überschreiten und können in manchen Ländern nur einmal um eben diese Frist verlängert werden. Ausländische Arbeitskräfte unterliegen üblicherweise der gleichen Beschränkung; in Obervolta beträgt die Höchstdauer der Ausländerbeschäftigung jeweils drei Jahre, in Senegal 30 Monate. Sieht ein Zeitvertrag die Möglichkeit fristgemäßer Kündigung vor, so handelt es sich nach insoweit einhelliger Rechtsprechung in Wahrheit um ein Arbeitsverhältnis von unbegrenzter Dauer ${ }^{44}$.

Wie bereits im Code du travail von 1952 geregelt, dürfen Arbeitsverträge auf Probe außer an der Elfenbeinküste $\mathbf{4 5}$ für höchstens sechs Monate abgeschlossen werden, mit ausländischen Arbeitskräften für längstens ein Jahr. Unbeschadet dieser Maximaldauer ist die Beschäftigung auf Probe an eine tarifvertraglich festgelegte Frist gebunden, die auf den Schwierigkeitsgrad der jeweiligen Tätigkeitsart abstellt und zwischen acht Tagen und sechs Monaten beträg ${ }^{\mathbf{4 6}}$. Im Interesse einer zuverlässigen Beurteilung des probeweise Eingestellten sind Arbeitsversäumnisse durch Krankheit und Unfall nicht auf die Probezeit anrechenbar. Wenn nichts Abweichendes vereinbart ist, kann jede Seite den Probevertrag fristlos kündigen, ohne daß dies eine Entschädigungspflicht zur Folge hätte. Einige neuere Gesetze verlangen freilich, daß der kündigende Vertragsteil seinen Schritt hinreichend begründet (Kongo) oder wenigstens, daß die Kündigung nicht rechtsmißbräuchlich ausgesprochen wird (Togo). Die stillschweigende Fortsetzung des Beschäftigungsverhältnisses über die vereinbarte Probezeit hinaus wird zuweilen (so in Senegal und Mauretanien) als rückwirkend gültiger Abschluß eines uneingeschränkten Arbeitsvertrages angesehen.

Wenn der Arbeitnehmer seinen Pflichten nicht nachkommt, bedeutet das nicht ohne weiteres eine Vertragsverletzung, sondern of tmals lediglich das zeitweilige Ruhen des Arbeitsverhältnisses (suspension du contrat de travail). Dies gilt z. B. bei Einberufung zum Militärdienst, Streik und Aussperrung, Krankheit und Unfall sowie bei außerordentlicher Beurlaubung ${ }^{47}$. Im Falle einer ärztlich attestierten Krankheit ruht das Arbeitsverhältnis bis zu sechs Monaten; die schwangere Frau erhält eine Dienstbefreiung von 14 Wochen. Der wegen Krankheit Arbeitsunfähige und der zum Wehrdienst Eingezogene bekommen in manchen Ländern (so die Elfenbeinküste, Niger, Obervolta, Benin) ihre bisherige Vergütung für die

\footnotetext{
43 Martin Kirsch, Le droit du travail africain Bd. II, Paris 1976, S. 29.

44 So etwa Cour suprême Gabon 16. 2. 1970: Travail et profession d'outre-mer Nr. 285, S. 6301.

45 Art. 32 Code du travail vom 1. 8. 1964, in: J. B. Blaise/M. Mourgeon, Lois e t décrets de Côte d'lvoire (Ed. Université d'Abidjan), Paris 1970 , S. $891 \mathrm{ff}$

46 Siehe z. B. für Senegal die „,Convention collective des journalistes professionnels“: Journal Officiel vom 11. 8. 1973, S. 1613 ff.

47 Dazu auch rechtshist orisch R. Mbella Mbappa, La rupture du contrat de travail à durée indétermirée au Cameroun, in: Recueil Penant 80 (1971), S. $59 \mathrm{ff}$.
} 
Zeit fortgezahlt, die der jeweiligen gesetzlichen Kündigungsfrist entspricht. Der berufstätigen Frau garantieren nur die Arbeitsgesetzbücher Malis, Benins und Guineas einen vollen Lohnausgleich während des Mutterschaftsurlaubs. Einhellig zugestanden wird dagegen das ungekürzte Arbeitseinkommen während des gesetzlichen Jahresurlaubs, dessen Umfang sich zwischen 18 (Tschad) und 26 (Kongo) Arbeitstagen beweg ${ }^{\mathbf{4 8}}$. Im übrigen ist die Höhe und Dauer der Lohnfortzahlung - so bei Arbeitsunfällen und Berufskrankheiten - durch die Vorschriften der Sozialgesetzgebung geregelt ${ }^{49}$.

\section{b) Beendigung des Arbeitsvertrages}

Die Vertragsauflösung nimmt in Gesetzgebung und Rechtsprechung einen breiten Raum ein. Auf unbestimmte Zeit abgeschlossene Arbeitsverträge können von jeder Seite unter Einhaltung einer Frist für beendet erklärt werden (ordentliche Kündigung). Bedarf diese Willenserklärung im allgemeinen der Schriftform, so lassen Obervolta, Guinea und die Elfenbeinküste die mündliche Kündigung ausreichen; allerdings kann der solcherart entlassene Arbeitnehmer innerhalb von acht Tagen eine schriftliche Bestätigung verlangen. In Kamerun, Niger, Tschad usw. muß der Grund für die Vertragsbeendigung in dem Kündigungsschreiben angegeben werden. Nach dem Gesetz Malis ist die Entlassung eines Beschäftigten, der für mehr als drei Monate eingestellt ist, an eine behördliche Genehmigung gebunden. In Senegal und Mauretanien hat der Arbeitgeber den Betriebsrat schriftlich zu benachrichtigen, wenn er Entlassungen zum Zwecke des Personalabbaus beabsichtigt.

Die Regelung der nach Tätigkeitsart und Dienstalter abgestuften Kündigungsfristen bleibt außer in Mali dem jeweiligen Tarifvertrag, hilfsweise einer Ausführungsverordnung überlas$\operatorname{sen}^{50}$. Wer sich ohne fristgerechte Kündigung aus einem Arbeitsverhältnis löst, ist dem anderen Vertragsteil zur Zahlung einer Entschädigung verpflichtet, deren Höhe sich nach dem vereinbarten Arbeitslohn und der restlichen Laufzeit des Kontraktes bemißt. Abweichend hiervon hat der entlassene Arbeitnehmer in einigen Ländern die Möglichkeit, bereits vor Ablauf der Kündigungsfrist ohne Rechtsnachteil eine neue Beschäftigung aufzunehmen, sobald er den bisherigen Arbeitgeber - in Kongo zwei Tage vorher - von dem geplanten Schritt unterrichtet hat.

Aus der Sicht der Gesetzgeber ist die Kündigung widerrechtlich, wenn sie mißbräuchlich (abusif), d. h. ohne rechtfertigenden Grund erfolgt (sans motif légitime) ${ }^{\mathbf{5 1}}$. Beispielhaft aufgeführt werden die Kündigung aus Anlaß von Meinungsäußerungen des Beschäftigten, aufgrund seiner gewerkschaftlichen Aktivitäten, wegen seiner Mitgliedschaft oder Nichtmitgliedschaft in einer bestimmten Gewerkschaft oder - so Kongo und Guinea - in einer politischen oder weltanschaulichen Gruppierung. Nach der Judikatur ist die Entlassung stets rechtswidrig, wenn sie sich auf einen der folgenden Tatbestände stützt: das außerdienstliche Verhalten des Arbeitnehmers, die Geltendmachung ihm zustehender Ansprüche, weit zurückliegende oder bereits geahndete Verfehlungen, die Weigerung der Leistung unbezahlter Uberstunden. Allgemein hat sich in der Rechtsprechung die Tendenz herausgebildet, die mit einem übergeordneten Interesse des Unternehmens, seiner wirtschaftlichen und technischen Organisation begründeten Kündigungen als legitim anzusehen. Dazu gehören etwa Entlas-

48 Es handelt sich um einen Mindesturlaub; tarifvertraglich kann eine längere Urlaubsdauer vereinbart sein. Dazu M. Kirsch Bd. II, a. a. O., S. $187 \mathrm{f}$.

49 P.-F. Gonidec, Cour de droit du travail, a. a. O., S. 265-270; E. Ehrentraut, a. a. O., S. $108 \mathrm{ff}$

$50 \mathrm{Vgl}$. Cour suprème Sénégal 24. 4. 1968: Travail et profession d'outre-mer Nr. 310, S. 6853.

51 Vgl. allgemein Roger Doublier, Manuel de droit du travail du. Cameroun, Paris 1973, S. 217 ff. mit weiteren Nachweisen. 
sungen wegen Verschlechterung der Auftragslage ${ }^{52}$, wegen einer Umstrukturierung des Unternehmens, die über eine bloße Änderung der Eigentumsverhältnisse hinausgeht ${ }^{53}$, wegen mangelhafter Leistungen des Arbeitnehmers oder grober Verstöße gegen die innerbetriebliche Ordnung. Als Kündigungsgründe, die in der Person oder dem Verhalten des Beschäftigten liegen, sind namentlich anerkannt: unentschuldigtes Fernbleiben von der Arbeit, eigenmächtige Verlängerung des Urlaubs, die Verrichtung betriebsfremder Tätigkeiten während der Dienstzeit, Arbeitsverweigerung bzw. Aufsässigkeit und die physisch bedingte Dienstunfähigkeit ${ }^{54}$. Bei Entlassungen zum Zwecke der Personaleinsparung verpflichten einige Arbeitsgesetzbücher den Unternehmer, die fachliche Eignung, das Dienstalter und die soziale Lage der Belegschaftsmitglieder zu berücksichtigen und den Betriebsrat von dem Vorhaben schriftlich in Kenntnis zu setzen. Auch haben die von einem Personalabbau Betroffenen mancherorts während einer bestimmten Frist - in Mauretanien ein Jahr - das Recht auf bevorzugte Wiedereinstellung. Von seiten des Beschäftigten ist die ordentliche Kündigung immer dann statthaft, wenn sie aus persönlichen oder familiären Motiven erfolgt oder wenn der Betreffende sich von einem Arbeitsplatzwechsel finanzielle Vorteile verspricht ${ }^{55}$.

Jedes Arbeitsverhältnis, ob zeitlich unbegrenzt oder begrenzt, kann durch außerordentliche Kündigung fristlos beendet werden, wenn der andere Vertragsteil sich einer groben Pflichtverletzung (faute lourde) schuldig macht. Als schwere Verfehlungen in diesem Sinne, deren Vorliegen das zuständige Gericht jeweils feststellen muß, gelten etwa die Begehung bestimmter Straftaten, die Mißachtung von Sicherheitsvorschriften, die Vorenthaltung des Lohnes und die physische Úberforderung des Arbeitnehmers ${ }^{56}$.

\section{c) Entschädigungsregelungen}

Eine Schadenersatzpflicht besteht insbesondere in den Fällen nicht fristgerechter sowie mißbräuchlicher Kündigung, zuweilen auch generell im Falle einer dem Arbeitnehmer nicht vorwerfbaren Vertragsauflösung, ferner bei unterbliebener oder unzureichender Ausstellung eines Arbeitszeugnisses. Daß und inwieweit eine Vertragspartei-sich durch Nichteinhaltung der Kündigungsfrist haftbar macht, wurde bereits ausgeführt. Größere praktische Bedeutung hat die Verantwortlichkeit dessen, der sich ohne anerkannten Grund einseitig von der vertraglichen Bindung löst. Die Entschädigung erfolgt nicht etwa durch Zahlung eines Pauschalbetrages, sondern durch den Ausgleich aller tatsächlich eingetretenen Nachteile, über deren Existenz und Umfang das angerufene Gericht anhand verschiedener gesetzlicher Kriterien entscheidet ${ }^{57}$. So werden bei der Ermittlung des durch eine Entlassung verursachten Schadens neben der Art der verrichteten Tätigkeit auch das Lebensalter des Beschäftigten, sein Dienstalter und seine wohlerworbenen Rechte (droits acquis) berücksichtigt. Anders als in Senegal und Kongo, wo der Arbeitgeber den gegen ihn erhobenen Vorwurf mißbräuchlicher Kündigung gegebenenfalls entkräften muß, hat der Arbeitnehmer grundsätzlich alle anspruchsbegründenden Tatsachen zu beweisen ${ }^{58}$.

Neben diesen Kündigungsfolgen kann der ohne eigenes Verschulden entlassene Arbeitnehmer nach den Gesetzen Malis und der Elfenbeinküste eine Abfindung verlangen (indemnité

52 Cour suprême Togo 6. 7. 1972: Travail et profession d'outre-mer Nr. 346, S. 7647

53 Cour d'appel Dakar 9. 7. 1969: Travail et profession d'outre-mer Nr. 271, S. 5995.

54 Cour d'appel Bangui 29. 11. 1967: Travail et profession d'outre-mer Nr. 253, S. 5613

55 Cour d'appel Dakar 9. 12. 1964: Travail et profession d'outre-mer Nr. 166, S. 3677.

56 Dazu im einzelnen Martin Kirsch, Mémento du droit du travail outre-mer (numéro spécial du Recueil Penant), Paris 1968 , S. 83 ff.

57 In Kongo hat der ungerechtfertigt entlassene Arbeitnehmer primär einen Anspruch auf Wiedereinstellung.

58 So etwa Tribunal du travail Niamey 27. 12. 1972: Travail et profession d'outre-mer Nr. 367, S. 8113. 
de licenciement), deren Höhe sich nach seiner in dem betreffenden Unternehmen abgeleisteten Beschäftigungszeit bemißt. Einigkeit besteht über die Entschädigungspflicht des Arbeitgebers im Falle der Verweigerung oder unsachgemäßen Abfassung eines Zeugnisses nach Beendigung des Arbeitsverhältnisses (certificat de travail). Uber seinen regelmäßigen Inhalt hinaus - Beginn, Ende und Gegenstand der Beschäftigung - ist das Zeugnis in manchen Ländern auf weitere Einzelheiten auszudehnen. So fordern Guinea und Gabun auch Auskünfte über die Tätigkeitsgruppe und Gehaltsklasse des Arbeitnehmers (catégorie professionnelle). In Senegal darf die Beurteilung weder falsche noch tendenziöse, in Guinea keinerlei ungünstige Angaben über den Beschäftigten enthalten. Bei geringfügigen Mängeln des erteilten Zeugnisses hat der Betroffene zuweilen ausschließlich einen Anspruch auf Richtigstellung59.

\section{Der Tarifvertrag (convention collective)}

Die Arbeitsgesetzbücher definieren den Tarif- oder Kollektivvertrag ${ }^{60}$ als eine Úbereinkunft zur Regelung von Arbeitsbedingungen, abgeschlossen zwischen Repräsentanten einer oder mehrerer Gewerkschaften oder Arbeitnehmer-Berufsverbänden einerseits und einem oder mehreren Arbeitgeberverbänden oder Einzelunternehmern andererseits. Togo präzisiert diese Begriffsbestimmung dahin, daß der Tarifvertrag ,, die Eingehung der individuellen Beschäftigungsverhältnisse regelt, die Rechte und Pflichten der Parteien fixiert und die Arbeitsbedingungen vereinheitlicht"61. Während etwa Madagaskar die Tariffähigkeit auf Arbeitnehmerseite den Gewerkschaften vorbehält, dehnt Kamerun dieses Recht auf die Spitzenorganisationen der Arbeitnehmer aus. Zur Form des Kollektivvertrages heißt es übereinstimmend, daß er in der offiziellen Landessprache, d. h. französisch abzufassen ist. Hinterlegungspflicht, Publikation, Inkrafttreten und die Möglichkeit eines späteren Beitritts zu den Abmachungen sind von Land zu Land unterschiedlich geregelt. Zur Geltungsdauer enthalten die Gesetze eine Vorschrift, nach der jeder Tarifvertrag innerhalb näher zu bestimmender Formen und Fristen auflösbar sein muß ${ }^{62}$. Zeitlich begrenzte Kontrakte können für höchstens fünf Jahre abgeschlossen werden und wirken nach Ablauf der vorgesehenen Dauer noch bis zu ihrer Kündigung fort, sofern keine abweichende Vereinbarung getroffen ist. Den geographischen Geltungsbereich ihrer Absprachen fixieren die Tarifpartner selbst (so ausdrücklich Mali), wobei sie freilich an die Verwaltungsstruktur des jeweiligen Staatsgebietes gebunden sind ${ }^{\mathbf{6 3}}$. Innerhalb dieser räumlichen Grenzen erfassen die Tarifverträge Westafrikas gewöhnlich alle organisierten Arbeitnehmer eines bestimmten Wirtschaftszweiges, auch soweit sie verschiedenen Berufssparten angehören ${ }^{64}$. Die kollektivvertraglichen Regelungen treten allerdings nur dann an die Stelle einzelvertraglicher Vereinbarungen, wenn sie weder zuungunsten der Beschäftigten von dem bestehenden Gesetzeszustand abweichen (Günstigkeitsprinzip) noch gegen eine Vorschrift des ordre public verstoßen. Vorgesehen ist die Möglichkeit einer Erweiterung der Tarifbindung (extension de la convention collective) durch ihre Erstreckung auf nichtorganisierte Beschäftigte und Arbeitgeber

59 Tribunal du travail Tananarive 3. 4. 1971: Travail et profession d'outre-mer Nr. 322, S. 7128

60 Allgemein P.-F. Gonidec, Le droit et la pratique des conventions collectives de travail au Congo, in: Recueil Penant 79 (1969), S. 279-303 sowie S. 429-442.

61 Journal Officiel de la République Togolaise vom 10. 5. 1974, S. 8 (Art. 67).

$62 \mathrm{Vgl}$. Art. 4 der Convention collective des journalistes du Sénégal, a. a. O., S. 1614

63 Sodie entsprechenden Vorschriften Senegals, Obervoltas und der Elfenbeinküste; Nachweise bei M. Kirsch Bd. I, a. a. O., S. 337 ff

64 Bureau International du Travail, Les relations professionnelles dans certains pays africains, Genf 1964, S. 64. 
der betreffenden Branche oder Berufsgruppe ${ }^{65}$. Solche allgemeinverbindlichen Tarifverträge sind wegen ihrer weitreichenden Wirkungen an zusätzliche Voraussetzungen geknüpft. So erklären die Gesetzgeber in dieser Hinsicht nur die ,,repräsentativen“ "Gewerkschaften und Arbeitgeberverbände für tariffähig ${ }^{66}$, ohne indes für diese Eigenschaft einheitliche Kriterien aufzustellen. Maßgebend sind kraft Gesetzes vor allem das Ergebnis der Betriebsratswahlen, der Mitgliederbestand, die Höhe der Beiträge und die Unabhängigkeit der arbeitsrechtlichen Organisation, in Ländern wie Mauretanien und Niger außerdem deren einschlägige Erfahrungen und das Ausmaß der entfalteten Aktivitäten. Úber den repräsentativen Charakter der Vereinigung ergeht in allen Staaten eine Entscheidung des Arbeitsministers, die außer in Mali durch Rechtsmittel angefochten werden kann, sei es vor dem Verwaltungsgericht (Guinea, Kongo), beim Regierungschef (Mauretanien, Obervolta) oder beim Staatspräsidenten (Niger).

Zum obligatorischen Inhalt allgemeinverbindlicher Tarifverträge gehören Vorschriften über die Koalitions- und Meinungsfreiheit der Beschäftigten, die Regelung des Arbeitsentgelts, Lohnzulagen für schwere und gefährliche Tätigkeiten, die Verwirklichung des Grundsatzes „,gleiche Arbeit - gleicher Lohn“, Dauer und Beendigung der Probezeit, die Regelung der Mitbestimmung, die Gewährung bezahlten Urlaubs und die Möglichkeit der „,̈nderung und der Auflösung des Tarifvertrages selbst ${ }^{67}$. Die Kodifikationen der einzelnen Staaten weisen diesbezüglich mancherlei Unterschiede auf. So verlangt Guinea von den Tarifpartnern auch Vereinbarungen über die Einstellungs- und Kündigungsbedingungen, das Schlichtungsverfahren bei Kollektivstreitigkeiten, die Durchführung der Lehrlingsausbildung und die besonderen Arbeitsbedingungen für Frauen und Jugendliche. Senegal und Togo gestatten eine Erweiterung des Katalogs durch Absprachen über Dienstalterszulagen, Leistungsprämien und Gewinnbeteiligungen. Nicht selten kann der Tarifvertrag auf weitere Punkte ausgedehnt werden, wofür die meisten Gesetze eine Reihe von Beispielen aufführen. Die Initiative für eine Allgemeinverbindlicherklärung liegt bei den repräsentativen arbeitsrechtlichen Koalitionen oder beim Arbeitsminister, auf dessen Beschluß hin eine paritätisch besetzte Tarifkommission zusammentritt und den Vertragsinhalt festlegt. Uber die Allgemeinverbindlichkeit der getroffenen Vereinbarungen entscheidet in aller Regel der Arbeitsminister, nachdem er seinen Schritt mit verschiedenen Fachgremien abgestimmt hat, etwa mit dem Arbeitsberatungsausschuß (Madagaskar, Kamerun) oder mit dem Nationalrat für Arbeitsfragen (Zentralafrikanische Republik ${ }^{68}$, Kongo). In Mauretanien ${ }^{69}$, Niger und Tschad kann ein solcher Tarifvertrag mehrere Beschäftigungszweige zugleich erfassen, in Mali können einzelne Vertragsbestimmungen von der Allgemeinverbindlichkeit ausgenommen werden, und in Senegal wird ausdrücklich betont, daß der Kontrakt nicht ohne weiteres rückwirkende Kraft hat ${ }^{70}$.

\footnotetext{
65 Siehe etwa für Gabun die Convention collective des exploitations forestières, étendue le 4 janvier 1964: Journal Officiel vom 1. 2. 1964, S. 75.

66 In den Arbeitsgesetzbüchern ist die Rede von ,,les organisations syndicales les plus représentatives“.

$67 \mathrm{Vgl}$. den ausführlichen Katalog in Art. 70 des Coded u travail der Elfenbeinküste, abgedruckt bei J. B. Blaise/J. Mourgeon, a. a. O., S. $899 \mathrm{f}$.

68 Seit dem 4. 12. 1976: Zentralafrikanisches Kaiserreich („Empire Centrafricaine“).

69 So die Convention collective nationale vom 13. 2. 1974: Journal Officiel de la République islamique de Mauritanie vom 3. 5.1974 , S. $181 \mathrm{ff}$.

70 Keinesfalls kann die vereinbarte Rückwirkung einen Arbeitgeber binden, der vor der Allgemeinverbindlicherklärung nicht Tarifvertragspartei war (Urteil des Arbeitsgerichts Dakar vom 26. 1. 1960: Recueil Penant 70/1960, S. 242-244 mit Anm. P.-F. Gonidec).
} 


\section{Schlußbemerkung}

Wie aus den vorangehenden Darstellungen ersichtlich, folgen die westafrikanischen Kodifikationen weithin den Regelungen des französischen Übersee-Arbeitsgesetzbuches von 1952; als Vorbild dienten zum Teil auch die Úbereinkommen und Empfehlungen der International Labour Organisation, die zahlreich abgeschlossenen Tarifverträge und die Entscheidungen verschiedener Gerichte ${ }^{71}$. Besonders das jeweilige Arbeitsvertragsrecht knüpft eng -stellenweise sogar wörtlich - an die kolonialrechtlichen Vorschriften an, womit zugleich gesagt ist, daß zwischen den einzelnen Staaten keine tiefgreifenden Normenunterschiede bestehen. Ebenso deutlich wie das Recht des Einzelarbeitsvertrages ist das geltende Tarifvertragsrecht aus dem Code 1952 hervorgegangen. Die eingeführten Neuerungen sind oft lediglich formalrechtlicher Art, bedingt durch politische und administrative Veränderungen anläßlich der Entstehung unabhängiger Staaten. Soweit es sich um materiell Neues handelt, dient es vor allem der erhöhten Stabilität des individuellen Arbeitsverhältnisses. Beispiele für diesen zeitgemäßen sozialen Wandel sind die erwähnten Maßnahmen des Mutterschutzes und des allgemeinen Kündigungsschutzes, die beide auf eine Initiative der Internationalen Arbeitsorganisation zurückgehen ${ }^{72}$, der heute alle ehemals französischen Úberseegebiete als souveräne Staaten angehören.

$71 \mathrm{Zu}$ den Quellen des Arbeitsrechts P.-F. Gonidec, Cours de droit du travail, a. a. O., S. 23-66.

72 Grundsätzlich Egbert Fried, Rechtsvereinheitlichung im internationalen Arbeitsrecht. - Eine Untersuchung zur Methode der Rechtsvereinheitlichung am Beispiel der Internationalen Arbeitsorganisation, Frankfurt a. M./Berlin 1965, S. 45. 


\section{Principles of Labour Law in Francophone Africa}

BY GUNTER WIEDENSOHLER

In West and Central African territories labour law could not develop before 1920, when slavery was abolished. The event probably most important during that period of colonial jurisdiction was the proclamation in 1952 of the "Code du travail des territoires d'outre-mer" whose influence on the future independant states was considerable.

After independence the states in question, in their constitution, firmly established fundamental concessions such as the right to employment and holidays, freedom of association, freedom to strike, freedom to make individual labour contracts. In general, the duty to work is given attention too, but any type of forced labour is prohibited. As to the freedom of making contracts, the lawmaker decreed several exceptions to the law, in order to protect life and health of the employees (limitation of children's and women's labour as well as nightwork) and by authorizing the executive power to intervene in the labour market for political and structural reasons.

The protection against dismissal which both, constitution and courts are giving a special attention, is due to recommendations from the International Labour Organization. The period of notice is graduated according to the kind of activity and years of service, and is usually regulated by collective agreement. In the case of undue dismissals the employee has a right to an indemnification whose amount the tribunal will decide after considering all aspects.

Compared to the French Overseas Labour Code of 1952 the innovations have only a formal character and are the result of political and administrative changes in the process of independence. 